\title{
Trajectory Tracking Control Design of a Mass- Damping-Spring System with Uncertainty using the Bond Graph Approach
}

\author{
Ismail Dif \\ Electrical Engineering Department \\ LAADI Laboratory \\ University of Djelfa \\ Djelfa, Algeria \\ difismail@yahoo.fr \\ Khier Benmahammed \\ Ferhat Abbas University Setif 1 \\ LSI Laboratory \\ Setif, Algeria \\ khierben@ieee.org
}

\author{
Abdallah Kouzou \\ Electrical Engineering Department \\ LAADI Laboratory \\ University of Djelfa \\ Djelfa, Algeria \\ kouzouabdellah@yahoo.fr \\ Ahmed Hafaifa \\ Electrical Engineering Department \\ LAADI Laboratory \\ University of Djelfa \\ Djelfa, Algeria \\ hafaifa@hotmail.com
}

\begin{abstract}
This paper deals with the simulation, and design of a trajectory-tracking control law for a physical system under parameter uncertainty modeled by a bond graph. This control strategy is based on the inversion of the system through their causal Input/Output (I/O) path using the principle of bicausality to track the desired trajectory. The proposed control strategy is validated with the use of a simple mechanical mass-springdamper system. The results show that the bond graph is a very helpful methodology for the design of control laws in the presence of uncertainties. This proposed control can be applied in several applications and can be improved to ensure robust control.
\end{abstract}

Keywords-bond graph; trajectory-tracking; bicausality; inverse system; uncertainty; 20-sim simulation

\section{INTRODUCTION}

Bond graph modeling is a rapidly developing powerful tool for modeling multidisciplinary physical systems with graphic and algebraic characteristics that help analyzing system properties and synthesizing control laws or algorithms for system monitoring. The work presented in this paper is based on the application of the control law introduced in [1] on a system modeled by a bond graph which was improved by the study of parametric uncertainties introduced in $[2,3]$ and the work in [3] regarding incremental bond graphs for the derivation of the state model for the study of robustness of bond graph models. These uncertainties are included by adding some bond graph elements which describe their effect in the system, as described in Section IV. This work consists of the design of a trajectory-tracking control law using uncertain bond graph models, in the same way as they are used in the development of diagnostic algorithms for industrial systems [5-
7]. Therefore, this work can represent a foundation stone for the design of robust control laws using bond graphs to ensure the control of complex systems.

\section{BOND GRAPH MODELING}

The bond graph methodology for modeling physical systems was first introduced in 1961 [10] and was formalized in $[11,12]$. It is based on the principle of conservation of energy between interconnected physical systems. It formalizes the transfer of power in different parts of the physical system, which is represented by a graphical link of half-arrow (Figure 1 ), which is modeled by the product of two conjugate variables that form the power, the effort, and the flux. All phenomena in a physical system can be modeled by their characters: A power producer or active elements as effort source "Se" or flow source "Sf", power consumers or passive elements such as resistances with "R", energy storages with "I" or "C", power transformation with direct transformation, ("TF") or mixed transformation ("GY" or Gyrator), conservative power ("0" junction for common effort or " 1 " junction for common flow).

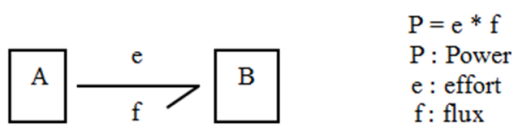

Fig. 1. Power transfer between systems A and B.

The bond graph modeling of such physical systems always goes through the following steps:

- The word bond graph, which is the abstract modeling of the system, dividing it into sub-systems interconnected by 
graphic links.

- The causal bond graph model is the representation of physical phenomena by bond graph elements, with connecting elements in the directions of power transfer.

- The causality application is the mathematics of bond graph modeling. It is the representation of cause and effect between interconnected systems. It is represented with a perpendicular stroke to bond representing the direction that the effort is known (see Figure 2). It is covered through selected assignment rules [13].
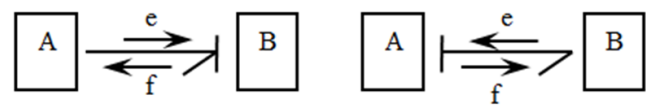

Fig. 2. Causality in bond graph.

As all modeling methods, the bond graph model allows its models to switch to other modeling forms such as block diagram, state space, or transfer function [13-14]. To better illustrate this methodology, the mechanical system and its bond graph model are presented in Figure 3.

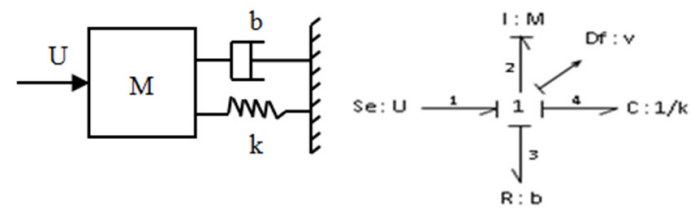

Fig. 3. Bond graph model of the mass-spring-damper system.

By following the bond graph modeling steps in Figure 3, the junction 1 shows the speed movement of the system, due to the force $U$ applied to the mass $M$ which is modeled by the inertia I, as system input or Se element. The output is represented by a flow detector Df, which is a speed sensor. The shock absorber and spring are modeled by $\mathrm{R}$ and $\mathrm{C}$ elements respectively. The concept of bicausality in a bond graph was introduced in 1995 [8]. It is used for the design of the inverse model of a system modeled by a bond graph. It is also used to estimate the parameters of such a model and/or to estimate its state evolution. In the causality concept, the causal stroke indicates the direction where the effort is known, while in the bicausality, the causal stroke is divided into two strokes, top and bottom, perpendicular to the two sides of the bond. The top line indicates the orientation of effort and flow, while the lower line indicates the source of the effort and flow. Figure 4 shows the difference between causality and bicausality.

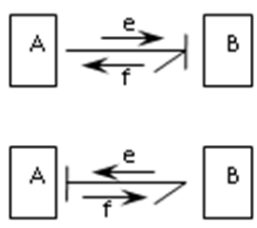

(a)

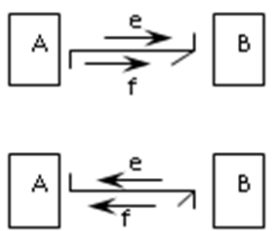

(b)

\section{TRAJECTORY-TRACKING CONTROL BY BOND GRAPH}

The synthesis of a control law is generally based on a model describing the behavior of the system under study. Physical systems vary from linear [15] to non-linear [16] and may or may not vary in time with several control techniques to regulate or track trajectory, leading to several control laws which may be linear such as the LQR control [17], LQR control based on bond graph model [18], PID and Intelligent PID control [19], and nonlinear control in several techniques based on graphical model (bond graph) [20-21], mathematical model (CTC, VSC, backstepping, $\mathrm{H}_{\infty}$, MPC, etc.) [22-24], or intelligent and meta-heuristic model optimization [19, 25-26], etc. [27-28]. In this paper, the tracking trajectory law is based in the technique initiated in [1] by the application of an algorithm of 5 steps which allows finding the control law for each junction block along with the causal I/O path. The algorithm steps are grouped in Table I.

TABLE I. TRAJECTORY -TRACKING LAW CONTROL DESIGN STEPS

\begin{tabular}{|c|c|c|}
\hline Steps & Step name & Operations \\
\hline Step 1 & Causal inversion & $\begin{array}{c}\text { Inversion of I/O minimal causal dynamic path in } \\
\text { the bond graph model using the bicausality }\end{array}$ \\
\hline Step 2 & Inverse equation & $\begin{array}{c}\text { Derivation on the bond graph model of the } \\
\text { minimum dynamic equation I/O }(m d e) \text {, which } \\
\text { presents the error }\end{array}$ \\
\hline Step 3 & $\begin{array}{c}\text { Output } \\
\text { substitution }\end{array}$ & Output substitution in $m d e: y(t)=y^{*}(t)-e(t)$ \\
\hline Step 4 & Error dynamics & Constraining the error dynamic: $\dot{e}(t)=-k^{*} e(t)$ \\
\hline Step 5 & Error substitution & Error substitution by: $e(t)=y^{*}(t)-y(t)$ \\
\hline
\end{tabular}

By applying the above procedure on the bond graph model of Figure 3, the steps can be described as follows:

Step 1: Bond graph inversion, which is to build the inverse $\mathrm{I} / \mathrm{O}$ model along the causal I/O path, as shown in Figure 5.

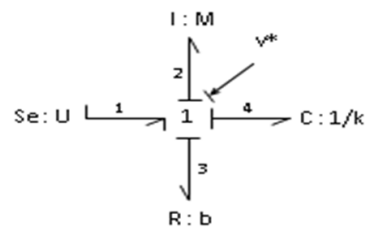

Fig. 5. Inverse bond graph model.

Step 2: Derivation of the (mde) following the inversion equation. The virtual input is obtained as:

$$
U=b \cdot v+M \cdot \frac{d v}{d t}+\frac{1}{C} \int v d t
$$

Step 3: Output substitution. The error of the output is defined as:

$$
e_{v}=v^{*}-v
$$

This leads to:

Fig. 4. Bond graph (a) causality vs. (b) bicausality. 


$$
U=b\left(v^{*}-e_{v}\right)+M \cdot\left(\dot{v}^{*}-\dot{e}_{v}\right)+\frac{1}{C} \int\left(v^{*}-e_{v}\right) d t
$$

Step 4: Error dynamics. The dynamics of the error is defined as a 1 st order equation (integrator):

$$
\dot{e}_{v}+k_{v} e_{v}=0, \quad \text { with } \quad k_{v}>0
$$

where (3) becomes:

$$
U=b\left(v^{*}-e_{v}\right)+M \cdot\left(\dot{v}^{*}+k_{v} e_{v}\right)+\frac{1}{C} \int\left(v^{*}-e_{v}\right) d t
$$

Step 5: Error substitution. Using (2) into (5) yields (6):

$$
U^{*}=\left(b-M k_{v}\right) v+\frac{1}{C} \int v d t+M \cdot \dot{v}^{*}+M \cdot k_{v} \cdot v^{*}
$$

Equation (6) is the relationship of the control input, so that the output $v$ follows the reference $v^{*}$, with a well appropriate choice of constant $k_{v}$ for a better solution. The application of this technique was done with the utilization of 20-sim [9] version 2.3 which allows the simulation of systems modeled by bond graphs. Using the available toolboxes, the bond graph model of the presented system is implemented (Figure 4) and with the control law developed by (6), the graph in Figure 6 is obtained for speed with square trajectory and a choice of $k_{v}=200$. The graph in Figure 6 shows that the system tracks well the desired path with the proposed control technique. In the next section, the uncertainty modeling parameters in a bond graph model are introduced and the control law for the uncertain model is recalculated.

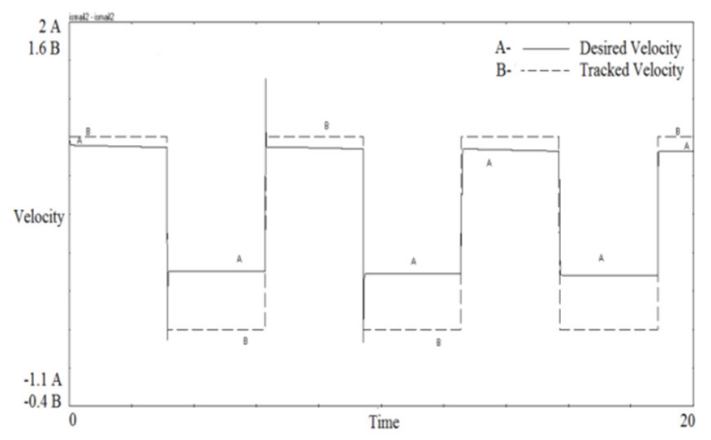

Fig. 6. Tracking-trajectory control simulation.

\section{UNCERTAINTY IN BOND GRAPH}

The bond graph tool by its graphical character and its analytical and structural performances are also used to represent modeling uncertainties as action elements on the system bond graph model. The introduction of uncertainty addition [2, 3] was followed by [4]. This technique can be applied to the design of robust diagnosis algorithms of bond graph models [5-7]. In the literature, two types of consideration can be found on the study of uncertainties: structured and unstructured uncertainties [29]. The current work is focused in the second type, which includes modeling uncertainties of physical phenomena, such as additive and multiplicative uncertainty at the input or the output. In bond graph modeling, both types of uncertainty (additive and multiplicative) are studied in [3], whereas in this work, the effect of input multiplicative uncertainty in the synthesis of control law is proposed. This is done using the standard interconnection model shown in Figure 7.

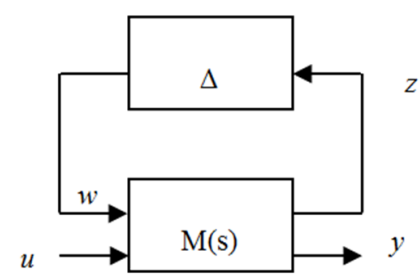

Fig. 7. Standard interconnection model.

The standard interconnection model can be represented by the following system of equations:

$$
\left\{\begin{array}{l}
\dot{x}=A x+B_{1} w+B_{2} u \\
z=C_{1} x+D_{11} w+D_{12} u \\
y=C_{2} x+D_{21} w+D_{22} u
\end{array}\right.
$$

where $x$ is the state vector, $u$ the input vector, $w$ the auxiliary input vector, $z$ the auxiliary output vector, $y$ the output vector, $\mathrm{M}(\mathrm{s})$ is the nominal system, and $\Delta$ the diagonal uncertainty matrix, where $w=\Delta . z$ with $\Delta=\operatorname{diag}\left(\delta_{i}\right)$ and $\delta_{i}$ is the $i^{\text {th }}$ parameter of the system.

In the bond graph model, only passive components $(\mathrm{R}, \mathrm{C}$, and I) are assumed and are subject to uncertainty with transformation elements (TF and GY). An example of the design of the standard interconnection model for the I element in integral causality, is presented as follows: the constitutive law of the I element in integral causality is:

$$
f=\frac{1}{I} \int e \cdot d t
$$

By introducing the term of the multiplicative error, it is found that:

$$
f=\frac{1}{I_{n}}\left(1+\delta_{I}\right) \int e . d t \text { With } \delta=-\frac{\Delta I}{I_{n}+\Delta I}
$$

The bond graph model of the uncertain element $I$ and in the last relation (9) gives the representation of Figure 8. By the addition of uncertainty on the I element of our model in Figure 3 , the model in Figure 9 is obtained.

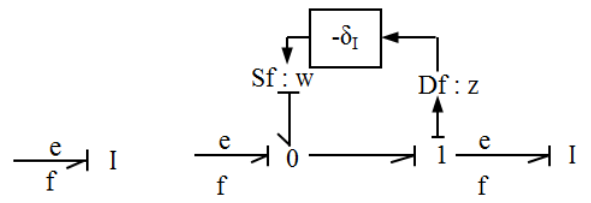

(a)

(b)

Fig. 8. Bond graph model of the element I: (a) nominal (b) with uncertainty. 


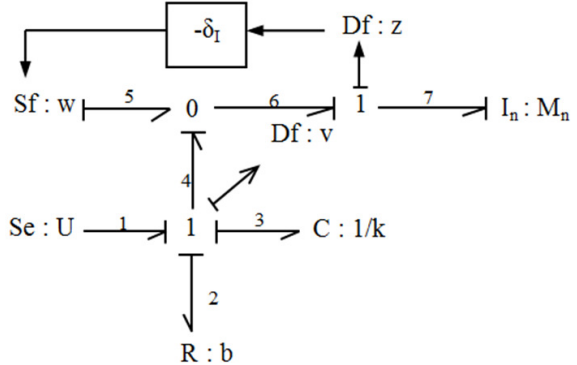

Fig. 9. Bond graph model with uncertainty.

\section{DESIGN OF THE CONTROL LAW}

By applying the control law design procedure stated above on the bond graph model with the uncertainty of the system presented in Figure 3, the following steps can be deduced.

Step 1: Bond graph model inversion. Applying the inversion procedure of the causal path input/output on our model, using the principle of bicausality, the following scheme is obtained:

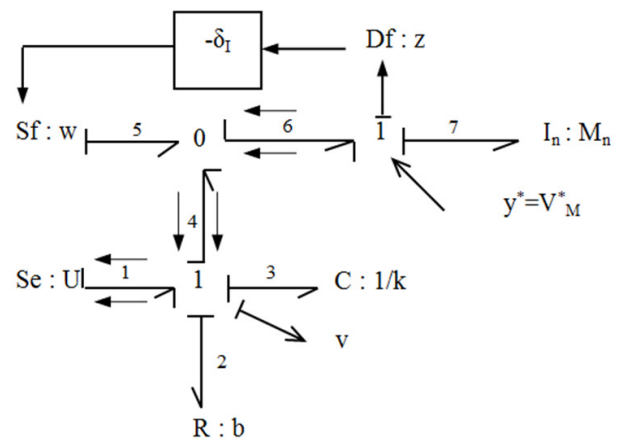

Fig. 10. Inverse I/O causal path.

Step 2: Derivation of the dynamic equation. Using the structural equations of junctions leads to:

- Junction 1: $v_{M}: e_{6}=e_{7}$ with $e_{7}=M_{n} \cdot \dot{v}_{M}$

- Junction $0: f_{4}=f_{6}-f_{5}$ with:

$$
\left\{\begin{array}{l}
f_{4}=v \\
f_{6}=f_{7}=v_{M} \\
f_{5}=-v_{M} \cdot \delta_{M}
\end{array}\right.
$$

Hence:

$$
v=\left(1+\delta_{M}\right) \cdot v_{M}
$$

- Junction $1: v e_{1}=e_{2}+e_{3}+e_{4}$ with:

$$
\left\{\begin{array}{l}
e_{1}=U \\
e_{2}=\frac{1}{C} \int v \cdot d t=k \int v \cdot d t \\
e_{3}=b \cdot v \\
e_{4}=e_{6}=e_{7}=M_{n} \cdot \dot{v}_{M}
\end{array}\right.
$$

Hence:

$$
U=b \cdot v+M_{n} \cdot \dot{v}_{M}+k \int v \cdot d t
$$

Replaces the speed $v$ using (10) and (12) takes the following form:

$$
U=b\left(1+\delta_{M}\right) v_{M}+M_{n} \cdot \dot{v}_{M}+k\left(1+\delta_{M}\right) \int v_{M} \cdot d t
$$

Step 3: Output substitution. The output error is defined as:

$$
e_{v_{M}}=v^{*}{ }_{M}-v_{M} \Rightarrow v_{M}=v^{*}{ }_{M}-e_{v_{M}}
$$

This leads to the relation:

$$
\begin{aligned}
U & =b\left(1+\delta_{M}\right)\left(v^{*}{ }_{M}-e_{v_{M}}\right)+M_{n}\left(\dot{v}_{M}^{*}-\dot{e}_{v_{M}}\right) \\
& +k\left(1+\delta_{M}\right) \int\left(v^{*}{ }_{M}-e_{v_{M}}\right) \cdot d t
\end{aligned}
$$

Step 4: Error dynamics. The dynamics of the error is defined as a 1 st-order equation (integrator):

$$
\dot{e}_{v_{M}}+k_{v_{M}} e_{v_{M}}=0 \text { with } k_{v_{M}}>0 \text { then } \dot{e}_{v_{M}}=-k_{v_{M}} e_{v_{M}}
$$

Equation (16) becomes:

$$
\begin{aligned}
U & =b\left(1+\delta_{M}\right)\left(v^{*}{ }_{M}-e_{v_{M}}\right)+M_{n}\left(\dot{v}_{M}^{*}+k_{v_{M}} e_{v_{M}}\right) \\
& +k\left(1+\delta_{M}\right) \int\left(v^{*}{ }_{M}-e_{v_{M}}\right) \cdot d t
\end{aligned}
$$

Step 5: Error substitution. Returning to (15) yields to (18):

$$
\begin{aligned}
U & =\left[b\left(1+\delta_{M}\right)-M_{n} k_{v_{M}}\right] v_{M}+M_{n}\left(\dot{v}_{M}^{*}+k_{v_{M}} e_{v_{M}}\right) \\
& +k\left(1+\delta_{M}\right) \int v_{M} \cdot d t
\end{aligned}
$$

which is the control law. The output $v_{M}$ follows the desired trajectory $v_{M}^{*}$.

To validate the proposed control for this system, the control relation (19) is implemented on the 20 -sim simulator, with a choice of nominalized values of parameters, and a value of $k_{v M}$ which allows reaching the control objective (tracking the trajectory of the desired speed), with a square-shaped signal, which for example simulates the motion of a rail traction chain. After choosing the uncertainty of the I element, which simulates additional load in a random form, the result shown in Figure 11 is found. The uncertainty of the presented case is chosen as random fluctuations bounded by the value $( \pm 0.5)$ around the normalized nominal value. Figure 11 shows the desired trajectory (curve B) followed by the output of our system (curve A), which confirms the efficiency of the designed control to the uncertainties of the system parameters. The error between the desired and the tracked velocity is around $10 \%$ from the nominal value, which is accepted in a control law. The contribution of this work is in the use of graphical and algebraic properties of the bond graph tool for the design of the control law, when the model is in presence of uncertainties, without the need for mathematical equation derivation, such as used in [18, 20-21]. 


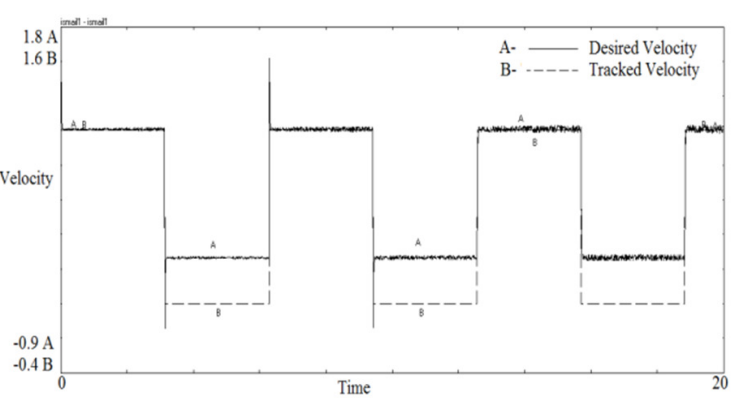

Fig. 11. Simulation result of designed control with 20-sim simulator

For the implementation of this control law, a platform is needed that integrates bond graph models, such as the 20 -sim software in later versions than the one used in this paper, which allow interaction with practical systems. We are working on the integration of bond graph models in embedded systems to use them in the design of control and diagnostic systems.

\section{CONCLUSION}

The main aim of this paper was to design a robust trackingtrajectory control system which considers the uncertainties of system parameters, based on bond graph modeling. It has been proved that the bond graph is a powerful tool for modeling a dynamic system to ensure the analysis of interconnected physical systems. It is also very efficient for the design of control laws. The simulation results show the effectiveness and the robustness of the proposed and designed controller against parametric changes of the presented application. On the other side, the proposed control algorithm can be improved to be a promising solution for several industrial control applications.

\section{REFERENCES}

[1] S. Junco, A. Donaire, and G. Garnero, "Speed control of series DC motor: a bond graph based backstepping design," in IEEE International Conference on Systems, Man and Cybernetics, Yasmine Hammamet, Tunisia, Oct. 2002, vol. 3, https://doi.org/10.1109/ ICSMC.2002.1176035.

[2] C. S. Kam and G. Dauphin-Tanguy, "Bond graph tools for standard interconnection structure determination," in Proceedings of the 2001 International Conference on Bond Graph Modeling and Simulation (ICBGM'01), Phoenix, AZ, USA, Jan. 2001.

[3] C. Sié Kam and G. Dauphin-Tanguy, "Bond graph models of structured parameter uncertainties," Journal of the Franklin Institute, vol. 342, no. 4 p. 379-399, Jul. 2005, https://doi.org/10.1016/ j.jfranklin.2005.01.005.

[4] W. Borutzky and G. Dauphin-Tanguy, "Incremental bond graph approach to the derivation of state equations for robustness study," Simulation Modelling Practice and Theory, vol. 12, no. 1, pp. 41-60, Apr. 2004, https://doi.org/10.1016/j.simpat.2003.11.002.

[5] M. A. Djeziri, B. Ould Bouamama, and R. Merzouki, "Modelling and robust FDI of steam generator using uncertain bond graph model," Journal of Process Control, vol. 19, no. 1, pp. 149-162, Jan. 2009, https://doi.org/10.1016/j.jprocont.2007.12.009.

[6] M. G. Kazemi and M. Montazeri, "A new robust fault diagnosis approach based on bond graph method," Journal of the Brazilian Society of Mechanical Sciences and Engineering, vol. 39, no. 11, pp. 4353-4365, Nov. 2017, https://doi.org/10.1007/s40430-017-0906-6.

[7] N. Tari, L. Sekhri, and H. Haffaf, "A Functional and Bond Graph Model for Robust Supervision," Electrotehnica, Electronica, Automatica (EEA), vol. 66, no. 1, pp. 161-171, 2018.

[8] P. J. Gawthrop, "Bicausal bond graphs," Simulation Series, vol. 27, 1994.
[9] ““'20-sim | Modeling \& Simulation,” 20-Sim. https://www.20sim.com/ (accessed Oct. 27, 2020).

[10] H. A. Paynter, Analysis and Design of Engineering Systems. The MIT Press, 1960.

[11] D. Karnopp, System dynamics: A unified approach. New York, NY, USA: Wiley, 1975.

[12] D. J. U. Thoma, Introduction to Bond Graphs and Their Applications: Pergamon International Library of Science, Technology, Engineering and Social Studies. Oxford, UK: Pergamon Press, 2016.

[13] P. Borne, G. Dauphin-Tanguy, J. P. Richard, F. Rotella, and F. Zambettakis, Automatique: Modélisation et identification des processus, tome 2. Paris, France: Editions Technip, 2000.

[14] G. Dauphin-Tanguy, Les Bond Graphs. Paris, France: Hermès, 2000.

[15] P. J. Antsaklis and A. N. Michel, A Linear Systems Primer. Boston, MA, USA: Birkhäuser, 2007.

[16] H. Khalil, Nonlinear Systems, 3rd Edition. Upper Saddle River, NJ, USA: Pearson, 2001.

[17] S. A. Ajwad, J. Iqbal, R. U. Islam, A. Alsheikhy, A. Almeshal, and A. Mehmood, "Optimal and Robust Control of Multi DOF Robotic Manipulator: Design and Hardware Realization," Cybernetics and Systems, vol. 49, no. 1, pp. 77-93, Jan. 2018, https://doi.org/ 10.1080/01969722.2017.1412905.

[18] M. Khemliche, I. Dif, S. Latreche, and B. O. Bouamama, "Modelling and analysis of an active suspension 1/4 of vehicle with bond graph," in First International Symposium on Control, Communications and Signal Processing, 2004, Hammamet, Tunisia, Mar. 2004, pp. 811-814, https://doi.org/10.1109/ISCCSP.2004.1296569.

[19] S. A. Ajwad, J. Iqbal, M. I. Ullah, and A. Mehmood, "A systematic review of current and emergent manipulator control approaches," Frontiers of Mechanical Engineering, vol. 10, no. 2, pp. 198-210, Jun. 2015, https://doi.org/10.1007/s11465-015-0335-0.

[20] T.-J. Yeh, "Controller synthesis for cascade systems using bond graphs," International Journal of Systems Science, vol. 33, no. 14, pp. 1161-1177, Jan. 2002, https://doi.org/10.1080/0020772031000071794.

[21] J. Zhao, D. Song, B. Zhu, Z. Chen, and Y. Sun, "Nonlinear Backstepping Control of Electro-Hydraulic Brake System Based on Bond Graph Model," IEEE Access, vol. 8, pp. 19100-19112, 2020, https://doi.org/10.1109/ACCESS.2020.2968513.

[22] J. Iqbal, M. I. Ullah, A. A. Khan, and M. Irfan, “Towards Sophisticated Control of Robotic Manipulators: An Experimental Study on a PseudoIndustrial Arm," Strojniski Vestnik - Journal of Mechanical Engineering, vol. 61, no. 7-8, pp. 465-470, https://doi.org/10.5545/svjme.2015.2511.

[23] S. Ullah, A. Mehmood, Q. Khan, S. Rehman, and J. Iqbal, "Robust Integral Sliding Mode Control Design for Stability Enhancement of Under-actuated Quadcopter," International Journal of Control, Automation and Systems, vol. 18, no. 7, pp. 1671-1678, Jul. 2020, https://doi.org/10.1007/s12555-019-0302-3.

[24] J. Iqbal, "Modern Control Laws for an Articulated Robotic Arm: Modeling and Simulation," Engineering, Technology \& Applied Science Research, vol. 9, no. 2, pp. 4057-4061, Apr. 2019, https://doi.org/10.48084/etasr.2598.

[25] M. Fouzia, N. Khenfer, and N. E. Boukezzoula, "Robust Adaptive Tracking Control of Manipulator Arms with Fuzzy Neural Networks," Engineering, Technology \& Applied Science Research, vol. 10, no. 4, pp. 6131-6141, Aug. 2020, https://doi.org/10.48084/etasr.3648.

[26] N. Dif, E. Boudissa, M. Bounekhla, and I. Dif, "Firefly Algorithm Improvement with Application to Induction Machine Parameters Identification," Revue Roumaine des Sciences Techniques - Serie Electrotechnique et Energetique, vol. 65, no. 1, pp. 35-40, 2020.

[27] W. E. Dixon, A. Behal, D. M. Dawson, and S. P. Nagarkatti, Nonlinear Control of Engineering Systems: A Lyapunov-Based Approach. Basel, Switzerland: Birkhäuser, 2003.

[28] J. Iqbal, M. Ullah, S. G. Khan, B. Khelifa, and S. Ćuković, "Nonlinear control systems - A brief overview of historical and recent advances," Nonlinear Engineering, vol. 6, no. 4, pp. 301-312, Dec. 2017, https://doi.org/10.1515/nleng-2016-0077.

[29] D. Alazard, P. Apkarian, C. Cumer, G. Ferreres, and M. Gauvrit, Robustesse et Commande Optimale. Toulouse, France: Cepadues, 1999. 